\title{
Review Article \\ Genetic, Functional, and Immunological Study of ZnT8 in Diabetes
}

\author{
Qiong Huang, ${ }^{1}$ Jie Du, ${ }^{1}$ Chengfeng Merriman, ${ }^{2}$ and Zhicheng Gong ${ }^{1}{ }^{1}$ \\ ${ }^{1}$ Department of Pharmacy, National Clinical Research Center for Geriatric Disorders, Xiangya Hospital, Central South University, \\ Changsha 410008, Hunan, China \\ ${ }^{2}$ Department of Physiology, Johns Hopkins School of Medicine, 725 North Wolfe Street, Baltimore, MD 21205, USA
}

Correspondence should be addressed to Zhicheng Gong; gongzhicheng@csu.edu.cn

Received 3 September 2018; Revised 14 November 2018; Accepted 5 December 2018; Published 26 February 2019

Academic Editor: Christian Goebl

Copyright (c) 2019 Qiong Huang et al. This is an open access article distributed under the Creative Commons Attribution License, which permits unrestricted use, distribution, and reproduction in any medium, provided the original work is properly cited.

Zinc level in the body is finely regulated to maintain cellular function. Dysregulation of zinc metabolism may induce a variety of diseases, e.g., diabetes. Zinc participates in insulin synthesis, storage, and secretion by functioning as a "cellular second messenger" in the insulin signaling pathway and glucose homeostasis. The highest zinc concentration is in the pancreas islets. Zinc accumulation in cell granules is manipulated by ZnT8, a zinc transporter expressed predominately in pancreatic $\alpha$ and $\beta$ cells. A common ZnT8 gene (SLC30A8) polymorphism increases the risk of type 2 diabetes mellitus (T2DM), and rare mutations may present protective effects. In type 1 diabetes mellitus (T1DM), autoantibodies show specificity for binding two variants of ZnT8 (R or W at amino acid 325) dictated by a polymorphism in SLC30A8. In this review, we summarize the structure, feature, functions, and polymorphisms of ZnT8 along with its association with diabetes and explore future study directions.

\section{Introduction}

Zinc is an essential ion for the body and maintains important biological functions acting as a catalyst, regulator, or structural component [1]. Intracellular zinc concentration is finely tuned by many proteins to maintain cellular homeostasis. The metallothionein (MT) family of zinc-binding proteins as well as zinc importers, known as ZIPs or SLC39 proteins, control the intracellular zinc uptake, while zinc transporters, known as ZnTs or SLC30 proteins, control the efflux of zinc into the extracellular matrix or intracellular vesicle $[2,3]$. Zinc functions as a "cellular secondary messenger" in the regulation of insulin signaling and glucose homeostasis [3], and zinc accumulation in granules is essential for insulin packaging, maturation, crystallization, trafficking, secretion, and regulation [4]. The concentration of zinc is about $30 \mathrm{mM}$ in granules of the pancreatic islet $\beta$ cells. Zinc is involved in the crystallization of insulin within secretory granules at pH5.5 until its secretion [5]. Preproinsulin is synthesized by ribosomes as a single-chain molecule, and the signal peptide is removed immediately to generate proinsulin [6]. Proinsulin is folded in the endoplasmic reticulum and binds with zinc to form a hexamer by electrostatic coupling at the acidic granules [7]. As the granules mature in the Golgi apparatus, proinsulin undergoes proteolytic processing which mediated by prohormone convertase to dissociate the C-peptide [6]. Six proinsulin monomers bind with two central zinc ions at histidine 30 in the B-chain to form a hexamer resulting in crystallized proinsulin. In contrast to the granules, the free cytosolic zinc concentration is only about $0.4 \mathrm{nM}$ [8], and it is important to maintain a high zinc gradient in the granules. If the zinc balance was disrupted, an abnormally high intracellular zinc concentration would cause cellular toxicity through thiol-dependent redox systems or by chelation of essential anions [4]. Diabetes is a metabolic disorder characterized by high blood glucose levels and decreased insulin secretion or increased insulin resistance $[9,10]$. Ninety percent of diabetes is type 2 diabetes mellitus (T2DM) [11]. Pancreatic $\beta$ cell dysfunction and/or insulin resistance are two major pathophysiological features of T2DM. Type 1 diabetes mellitus (T1DM) is an autoimmune disease which is caused by T cell-mediated destruction 


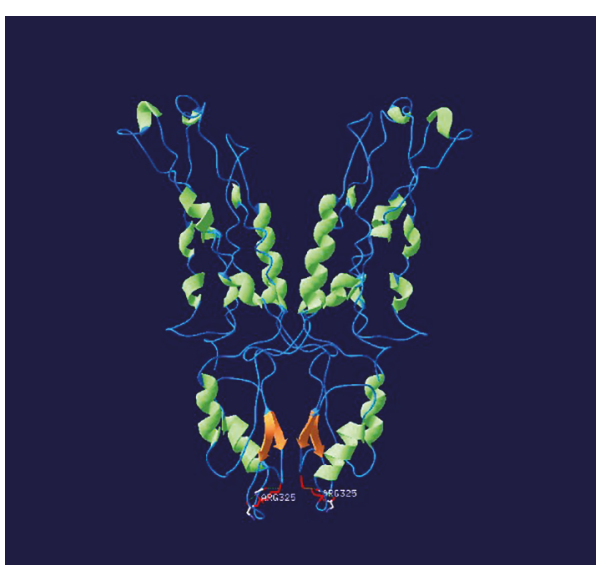

(a)

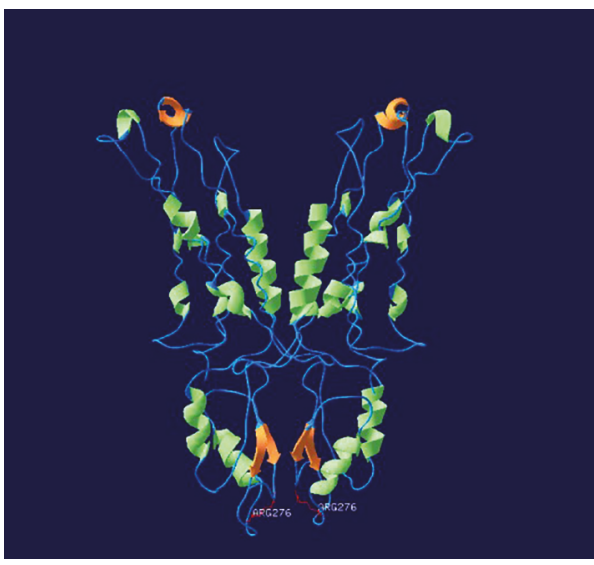

(c)

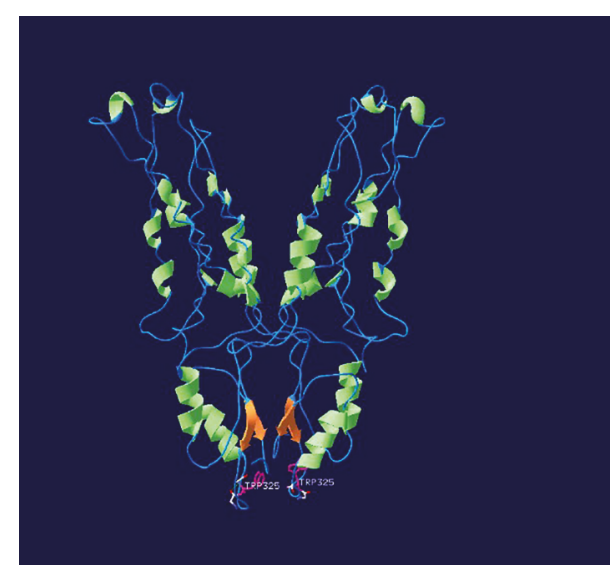

(b)

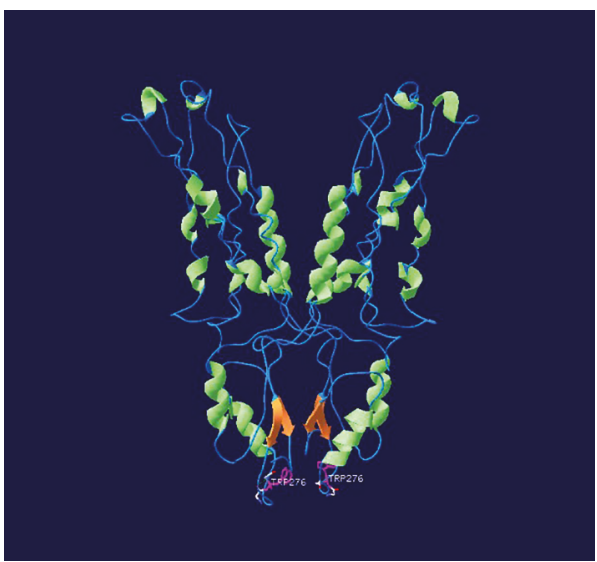

(d)

Figure 1: Homology model of the human ZnT8 homodimer with different forms and genotypes. The image in (a) is R325 in A form. The image in (b) is W325 in A form. The image in (c) is R325 in B form. The image in (d) is W325 in B form.

of the pancreatic $\beta$ cells [12]. Significantly decreased serum zinc levels and increased urinary zinc loss are characteristics of both T1DM and T2DM patients [13-15]. Some studies have shown that zinc supplement or high dietary zinc intake can reduce the risk of T2DM [16], which is modified by obesity and genotype effects [17].

Zinc transporter protein member 8 ( $\mathrm{ZnT} 8)$ is thought to be the regulator of zinc concentration in $\beta$ cells and also acts as a zinc sensor. ZnT8 is a unique protein as it is exclusively expressed in islets, and it facilitates the transport of zinc from the cytoplasm into secretory vesicles $[18,19]$. ZnT8 is associated with both T1DM and T2DM, as an antigenic target and a mediator of zinc enrichment in insulin secretory granules [2], respectively. In T1DM, the presence of ZnT8 autoantibodies is used as an important diagnostic tool. Genome-wide association studies (GWAS) reported an association of ZnT8 gene (SLC30A8) mutation with T2DM [20]. The risk single nucleotide polymorphism (SNP) rs13266634 was correlated to T2DM disease susceptibility in several populations. More recent studies focus on ZnT8 function, expression, and distribution, but there are some controversial or conflicting results regarding its function. This paper will review previous results as well as our studies to elucidate the role of $\mathrm{ZnT}$, its association with diabetes, and its future study directions.

\section{ZnT8 Features and Expression}

ZnT8 belongs to a subgroup of the cation diffusion facilitator (CDF) super family. It is a transmembrane protein composed of 369 amino acids in A form and 320 amino acids in B form, according to an N-terminal extension (Figure 1) [21]. Although the exact structure is not clear, ZnT8 is a dimermembrane protein and conforms to a "Y"-shaped structure similar to immunoglobulin architecture according to the bacterial YiiP X-ray structure [22, 23]. Each monomer consists of six transmembrane domains (TMDs) and a histidinerich loop with both the $\mathrm{N}$ - and C-terminals located in the cytoplasm [2]. ZnT8 acts as a $\mathrm{Zn}^{2+} / \mathrm{H}^{+}$exchanger [2] and pumps zinc ions from the cytosol into intracellular vesicles or into the extracellular space [21]. The antiport mechanism could be induced by conformational changes in the protein structure. There are four zinc-binding sites located in each protomer. The primary zinc transport site resides at the TMDs (site A), and the secondary site is located in the interface between the membrane and the cytoplasmic domain 
(site B) $[22,23]$. The C-terminal cytosolic domain has important functions in protein-protein interactions and as a zinc sensor [15].

$\mathrm{ZnT}$ 8 is presented in a cell- or tissue-specific manner. It is mainly expressed in the pancreatic islets and localized to the membrane of insulin granules of $\beta$ cells but can also be detected in pancreatic $\alpha$ cells [24]. At the mRNA level, ZnT8 is the zinc transporter with the highest expression in pancreatic tissues by far. At a level comparable to $\beta$-actin [25] or GAPDH [26], it has more than ten times the expression of other ZnT family members. This distribution pattern has been confirmed by a single-cell transcriptome study (RNA-Seq) of human pancreatic islets [27]. ZnT8 gene transcription is controlled by Pdx-1, a transcription factor enriched in $\beta$ cells [28]. ZnT8 seems to be cytokine sensitive as its expression can be downregulated by cytokines such as IL- $1 \beta$ and IFN- $\gamma$ [29]. In our previous localization studies, we used the stable expression of human ZnT8 in the INS-1E cell line to show specific antibody binding to the protein extracellular surface. We found abundant cell surface staining of ZnT8 and its coupling to glucose-stimulated insulin secretion (GSIS) which demonstrated the potential of $\mathrm{ZnT}$ 8 as a biomarker for tracking and isolating functional $\beta$ cells in mixed cell populations [30]. There have been many efforts to find $\beta$ cell surface markers with cell sorting potential $[31,32]$, and ZnT8 may prove to be a useful marker. ZnT8 can act in imaging, isolation of live $\beta$ cells from a heterogeneous population, or selection of differentiated functional $\beta$ cells from progenitors [30].

\section{ZnT8 Genetic Study in Human}

ZnT8 genetic studies mainly focus on mutations in its coding gene SLC30A8 which are associated with the risk of T2DM. The high-throughput GWAS assay provided the wild C allele variant of rs13266634 in SLC30A8 associated with T2DM risk in French [33, 34], Finnish [34], and English populations $[35,36]$, and it has been replicated in other populations such as Asian [37], Chinese [38-44], Japanese [45-48], Singaporean [49], Korean [50, 51], Arab [52], Norwegian [53], Caucasian women [54], African-American [55], Pakistani [56], Tunisian [57], Mexican mestizo [58], Saudi Arabian [59, 60], Kazakh [61], Iranian [62], Mayan [63], Thai [64], and Greek-Cypriot [65]. The mutated T allelic frequencies in the Asian papulation were higher. Furthermore, the meta-analysis also reported that the rs 13266634 polymorphism is among the most confirmed genetic markers for T2DM [66-74].

Not only is the SNP associated with T2DM, but there is also a relationship of rs13266634 with a substantial set of metabolic traits. Carriers with the rs13266634 C allele showed a significant decrease in insulin secretion [75-82] or a reduced proinsulin to insulin conversion [83]. The risk allele also shows a relationship with insulin sensitivity [84] and glucose tolerance from the Quebec Family Study [54]. The rs13266634 polymorphism showed a significant association with impaired glucose metabolism or impaired $\beta$ cell function in Russian populations [75] and with HbAlc in Thai populations [64]. The risk $\mathrm{C}$ allele was correlated with lower acute insulin response to glucose, and it was also correlated with a lower disposition index in a Chinese study [82]. A common genetic risk C variant in SLC30A8 influences, to different extents, the development of impaired fasting glucose (IFG), the transition from IFG to $\mathrm{T} 2 \mathrm{DM}[77,80,85]$, and the impairment of glucose metabolism $[75,78]$.

The rs13266634 polymorphism is a nonsynonymous SNP which causes $\mathrm{C}$ to be substituted by $\mathrm{T}$ at base pair position 973 causing the amino acid to change from arginine (R) to tryptophan (W) at position $325(\operatorname{Arg} 325 \operatorname{Trp}, \mathrm{R} 325 \mathrm{~W})$. The odds ratio (OR) value of the $\mathrm{R}$ form of ZnT8 for the risk of having T2DM is about 1.2. Other SNPs in SLC30A8, such as rs3802177 and rs11558471 [86] located in the $3^{\prime}$ untranslated region as well as rs16889462, which changes amino acid 325 from arginine (R) to glutamine (Q) (Arg325Gln, R325Q) [87], were also significantly associated with T2DM. Polymorphisms rs3802177 and rs11558471 are in strong linkage disequilibrium with rs13266634 [42, 88]. These genetic studies have shown that higher total zinc intake may attenuate the glucose-raising effect as well as lower fasting glucose levels [42] of the rs11558471 variant. SNP rs11558471 was strongly associated with T2DM $(P=0.002, \mathrm{OR}=1.334,95 \% \mathrm{CI}=1.110$ to 1.602$)$ and moderately associated with diabetic nephropathy in a Malay population [89]. Sun et al. reported that rs11558471 was associated with higher circulating proinsulin and lower insulinogenic index [16].

Not all of the human studies can identify the link between the rs13266634 polymorphism and the risk of susceptibility to T2DM. Phani et al. [76] and Khan et al. [90] confirmed the association of rs13266634 with T2DM, but another two studies showed no detectable association [91, 92]. T2DM was not found in association with this polymorphism in studies reported among Mexican American Families [93] and in African Americans of European descent [94]. There are also some controversial study results in Indian populations. The inconsistencies may be due to racial differences, different sample selection criteria, or alternative assay detection methods. Another interesting result from Flannick et al. reported some loss-of-function mutations in the SLC3OA8 gene which could protect against the risk of T2DM [95]. These 12 rare protein-truncating variants were statistically associated with a $65 \%$ decrease in T2DM risk. The strongest protective association was observed in Iceland between p.Lys34SerfsX50 and measuring random glucose levels in nondiabetic subjects [95]. This interesting result implied that this allele could be a new target for antidiabetic drug therapy. Another nonsense variation, R138X (c.412 C>T), has shown significant association with T2DM $(\mathrm{OR}=0.46, P=0.012)$. Moreover, experiments with the recent SLC30A8 R138X knock-in mouse model showed that insulin secretion was increased under hyperglycemic challenge, although the mouse had normal body weight, glucose tolerance, and $\beta$ cell mass [26]. The phenotype could possibly be related to a decrease in mitochondrial gene expression and an increase in the expression of the voltage-gated proton channel Hv1 (Hvcn1) [26]. These data also provide evidences in favor of targeting ZnT8 as a possible therapy for T2DM. 


\section{ZnT8 Functional Studies In Vivo and In Vitro}

How does ZnT8 expression level affect target cell functions? In the INS-1E cells, the consequences of ZnT8 overexpression were an increase in zinc accumulation and an enhancement of GSIS compared with control cells [26]. Cells with downregulated ZnT8 not only showed a functional decrease in insulin content and secretion in response to hyperglycemic stimulus but also showed fewer dense-core vesicles via electron microscopy [96]. These data suggest that ZnT8 is a key $\beta$ cell functional protein for zinc accumulation and insulin secretion.

Genetic mouse models used to investigate the whole animal or cell-specific ZnT8-KO effect on metabolic traits have confusing results. Exon 1 [97-100], exon 3 [101, 102], or exon 5 [103] were deleted in C57BL/6J [100, 101, 103, 104] or a mixed species mouse $[97-99,102]$ (Figure 2). All the ZnT8-KO animals showed no significant changes in growth, body weight, gross anatomy, or behavior compared with respective WT mice. Nearly all the ZnT8-KO mice showed no change in islet size, number, cell position, islet insulin content, and $\alpha / \beta$ cell ratio $[98,99,101-103]$. However, all of the studies showed that islet zinc content decreased. Some studies showed that the islet structure changed [97-100, 103], and pale insulin "progranules" were detected $[97,99]$, which implied that ZnT8 is necessary for zinc accumulation in islet cells. GSIS studies in the isolated islets showed no change $[99,100]$, a decrease $[97,101,102]$, or an increase $[98,103]$. During in vivo studies, the fasting glucose levels showed no change $[97,99,101-103]$ or an increase [98]. The fasting insulin content also showed no change $[97,99,101]$ or a decrease $[98,100,102,103]$. The intraperitoneal glucose tolerance test (IPGTT) showed impaired glucose tolerance (IGT) $[97,98,100,101,103]$ or normal glucose tolerance $[99,102]$. Oral glucose tolerance test (OGTT) studies also showed controversial results from no change [101] to IGT [97]. Results from GSIS studies in vivo showed decreased [98, 103] or unchanged [97]. The variation of metabolic-related phenotypes in ZnT8-KO mice may result from using different methods and different animal genetic backgrounds. Another study using the combined deletion of SLC3OA7 and SLC3OA8 found that SLC30A8 is critical for GSIS because a significant decrease of GSIS can be detected in both SLC30A7/SLC30A8-KO but not in the SLC30A7 single-KO animal. These data unmask the function of ZnT8 in islets by the removal of ZnT7 and imply that ZnT8 may affect T2DM susceptibility through other tissues where it is expressed at low levels rather than through effects on pancreatic islet function [105]. In isolated human islets, the risk $\mathrm{C}$ allele does not affect ex vivo insulin secretion or SLC30A8 expression. It also does not affect ZnT8 protein expression which is correlated with that of insulin and glucagon secretion [66].

Diabetes is a metabolic disease, although the environment plays an important role in its pathogenesis and development. One study used a $\beta$ cell-specific ZnT8-KO mouse model and a whole ZnT8-KO mouse model with a high-fat, high-calorie diet to investigate the phenotype as measured by metabolic indicators. In the $\beta$ cell-specific models, measurements indicated no weight change but a decrease of insulin synthesis and secretion was noted in addition to glucose intolerance. In the whole ZnT8-KO mouse model, after maintenance on a high-fat feed, the animals were significantly obese, hyperglycemic, hyperinsulinemic, insulin resistant, and glucose intolerant. The researchers concluded that $\mathrm{ZnT}$ 8 is important to the whole body and not just in $\beta$ cells only. The mechanism of diet-induced obesity in the ZnT8-KO mouse contributed to the T2DM risk in a $\beta$ cell-specific and nonspecific manner [106]. More evidences for the role of ZnT8 in diabetes were determined by Tamaki et al., who found that ZnT8-KO mice had lower peripheral blood insulin levels, although they had hypersecretion of insulin from pancreatic $\beta$ cells relative to WT animals. The secreted insulin was cleared by the liver during its first passage which was confirmed by the C-peptide/insulin ratio in the circulation [103]. This may help to explain the phenotype of ZnT8 SNP rs13266634 after knowing that the hepatic clearance of insulin may be impacted. These data imply that ZnT8 function should be evaluated in vivo as well as at the cellular and molecular levels.

In an $\alpha$ cell-specific ZnT8-KO mouse, the level of glucose and insulin tolerance was normal. However, the female models showed lower glucose infusion rates during hypoglycemic clamp experiments and increased glucagon secretion compared with WT controls. When responding to lower glucose $(1 \mathrm{nM})$, islets isolated from $\alpha$ cell-specific ZnT8-KO mice secreted more glucagon than WT controls. This suggested that ZnT8 is important for glucagon secretion stimulated by lower glucose in a subset of $\alpha$ cells [24]. Alternatively, ZnT8 overexpression in $\alpha$ cells showed increased zinc accumulation in the cytoplasm and granules and impaired glucagon secretion in response to hypoglycemia [107]. These data provide further evidence that T2DM-associated polymorphisms in the SLC30A8 gene may act in part via alterations in glucagon release and suggest that ZnT8 activation may restrict glucagon release in some settings.

\section{Functional Mutation Studies}

It has been difficult to confirm the functional phenotype attributed to the ZnT8 transporter polymorphism in vivo. Some studies have used purified human ZnT8 amino acid 325 protein to detect the function changes. Parsons et al. purified the cytoplasmic domain (CTD) of ZnT8 with R325 (ZnT8cR) or W325 (ZnT8cW) encoding mutations. The results showed that $\mathrm{ZnT}$ 8cR was more thermostable than $\mathrm{ZnT}$ 8cW, and the ZnT8cW monomers showed higher affinity to zinc [21]. A limitation of these studies is that they examined only the CTD instead of the full-length protein to evaluate ZnT8 activity. In our previous work, we used a highly sensitive functional assay in vitro to compare the functional difference between ZnT8's R form and $\mathrm{W}$ form. We found that the $\mathrm{R}$ form showed more activity when we induced expression in HEK293 cells, 


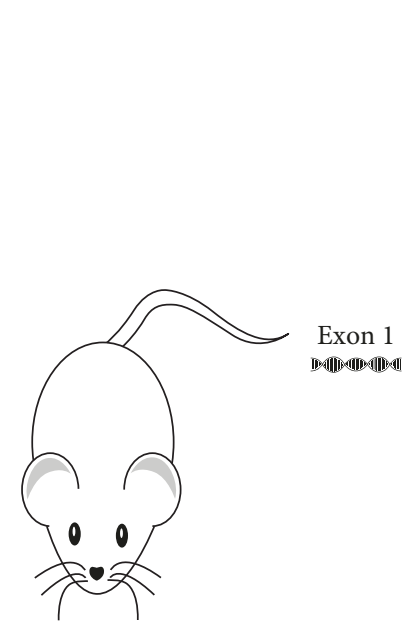

Mixed

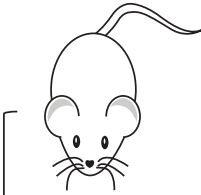

Whole body [98]

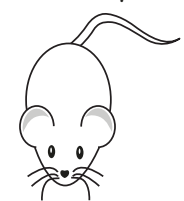

Whole body [99]

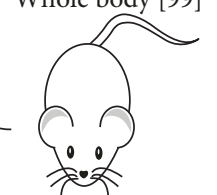

r

Specific (Ins1Cre) [97]

Exon 3

III. III AIII) IIII)

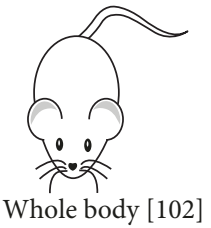

Whole body [102]

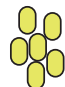

Unchanged

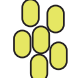

Unchanged

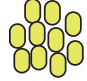

Increased

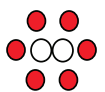

Rod-shaped cores

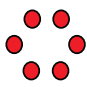

Loss of dense core granules

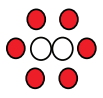

Rod-shaped cores

Unchanged

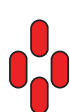

Not examined Not examined
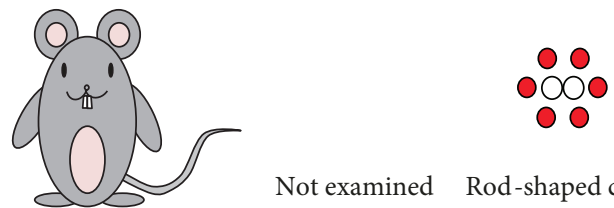

Not examined

Rod-shaped cores

Impaired

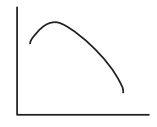

Normal

Impaired

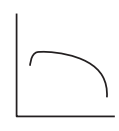

IGT

IS decreased

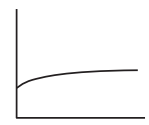

GSIS decreased

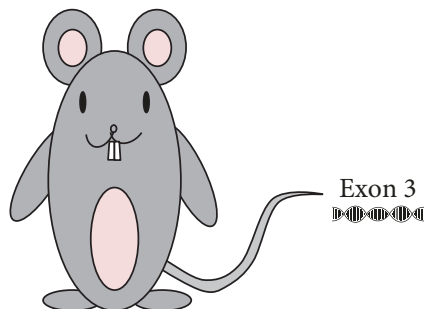

C57BL/6J
Exon 5 IIIIIIIIIIIIIIIII

Specific (Ins1Cre) [100]

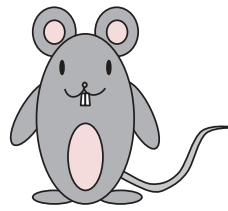

Whole body [101]

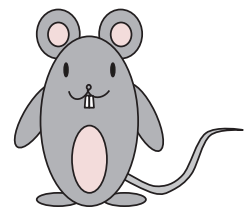

Specific (Ins2Cre) [103]

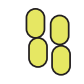

Decreased

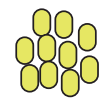

Increased

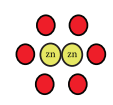

Unchanged

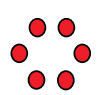

Loss of dense core granules

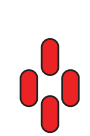

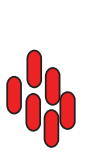

Unchanged

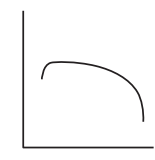

IGT

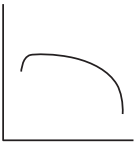

IGT

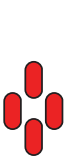

Impaired

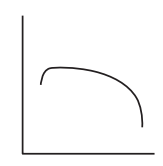

IGT

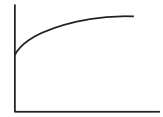

GSIS unchanged

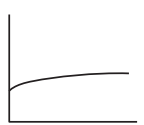

GSIS decreased

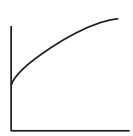

GSIS increased

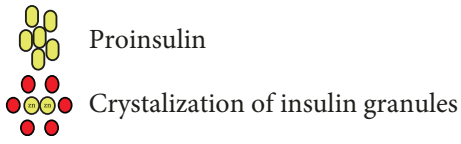

Insulin in vivo

IGT: impaired glucose tolerance

Figure 2: ZnT8 knockout mice in mixed genotypic mouse or C57BL/6J mouse showed a chaos phenotype.

purified ZnT8 in lipid remodeling, and measured using the stop flow method [21]. These results showed a higher activity of the ZnT8 R form which confers increased risk of T2DM, whereas the ZnT8 $\mathrm{W}$ form displays lower functional activity. Thus, ZnT8 has the potential to be a new target for protection against T2DM.

\section{ZnT8 and T1DMs}

T1DM is an autoimmune disease characterized by $\mathrm{T}$ cell-mediated selective destruction of $\beta$ cells in the pancreatic islets of Langerhans [108], the onset of which mainly occurs in childhood or young adulthood, and insulin is needed as 
an exogenous supplement. Autoantibodies including islet cytoplasmic autoantibodies (ICA), antibodies to insulin (IAA), the $65 \mathrm{kD}$ form of glutamate decarboxylase (GADA), and the protein tyrosine phosphatase IA2 (IA2A) can be used to identify the onset of or risk of developing T1DM in more than $80 \%$ of the patients $[108,109]$.

ZnT8 is a novel islet autoantigen for T1DM, and it has been shown that ZnT8 was targeted by autoantibodies in $60-80 \%$ of new-onset T1DM patients, which occurred in only $2 \%$ of controls, $3 \%$ of T2DM patients, and $30 \%$ of patients with other autoimmune diseases associated with T1DM [108, 110]. If combined with the measurement of GADA, IA2A, and IAA, autoimmunity detection rates would rise to $98 \%$ at disease onset $[111,112]$. It has also been confirmed that human ZnT8 autoantibodies showed no cross-reactivity with other ZnT family member proteins and no activation with mouse $\mathrm{ZnT} 8$ protein $[113,114]$. IAA is usually the first autoantibody to appear at a young age in the preclinical stage compared to anti-ZnT8 autoantibodies, which are often not detected before 3 years of age $[115,116]$. However anti-ZnT8 antibodies can detect up to $26 \%$ of T1DM patients who previously presented as autoantibody negative using IAA, ICA, or GADA assays [114]. Thus, anti-ZnT8 antibodies have been shown to exist in the blood from the prediabetic phase and are likely a downstream event of the primary genetic pathogenesis of T1DM [117]. It is a useful independent marker of autoimmunity either alone in antibody-negative subjects or combined with other T1DM autoantibody markers [115]. ZnT8 is a pancreatic-specific protein, and it could potentially be used to value the function of pancreatic islets.

ZnT8 is a multispanning transmembrane protein, and it is difficult to purify while maintaining its native structure. The existing antibody assays were preformed using radioimmunoprecipitation of the in vitro translation product from soluble CTD, N-domain, or fusion $\mathrm{N}$ - and C-terminal domains [108]. It is difficult to detect epitopes near transmembrane domains because no anti-TMD domain monoclonal antibody existed until now. Wan et al., through a combined bioengineering and nanotechnology approach, developed a proteoliposome-based full-length human ZnT8 antigen for efficient detection of a ZnT8 autoantibody on a plasmonic gold chip. This method showed efficient antiZnT8 antibody detection for T1DM diagnosis with about $76 \%$ sensitivity and $97 \%$ specificity [118]. The extra detection signal could come from autoantibodies targeting the extracellular surface(s) of ZnT8 in a pancreatic $\beta$ cell. The specificity of ZnT8 autoantibody reactivity was influenced by SNP rs13266634 in the new proteoliposome-based assay. T1DM patients with different genotypes, namely, CC, CT, or TT, exert different phenotypic reactivities with respective ZnT8 antigen proteins. Of the patients with the CC genotype, $42 \%$ had the $325 \mathrm{R}$ antibody, $32 \%$ had $325 \mathrm{R} / 325 \mathrm{~W}$ dual antibodies, and only $5 \%$ had $325 \mathrm{~W}$-specific antibodies [119]. TT carriers showed that $73 \%$ had the $325 \mathrm{R}$ antibody, $13 \%$ had $325 \mathrm{R} / 325 \mathrm{~W}$ dual antibodies, and none had 325W-specific antibodies. $90 \%$ of the CT subjects showed a reaction with either $325 \mathrm{R}$ or $325 \mathrm{~W}$-specific antibodies and, recently, a subclass of serum anti-ZnT8 antibodies polyclone directed to the surface of live [120] and isolate-specific monoclonal anti-ZnT8 [121]. So ZnT8 is important for T1DM diagnosis, and its gene mutations also affect the autoantibody reactivity.

\section{Summary}

In this paper, we reviewed studies of the structure, expression, and function of $\mathrm{ZnT}$. Scientists appreciate that ZnT8 plays an important role in zinc accumulation in pancreatic islets. Controlling the zinc concentration is important not only in the intracellular compartment but also in its subcellular redistribution to vesicles. Previously, studies expressing ZnT8 in $\beta$ cells demonstrated the substantial presence of ZnT8 and it has been difficult to distinguish the cytoplasmic versus the zinc granule distribution. Some studies focused on the SLC30A8 gene mutations and the efficacy of antidiabetic drugs. We found that SLC30A8 rs13266634 and rs16889462 polymorphisms were associated with the therapeutic efficacy of the antidiabetic drug repaglinide in Chinese T2DM patients [87]. In a diabetes prevention program study, 3007 subjects treated with metformin or troglitazone coupled with lifestyle changes for 1 year were genotyped and the results have shown that a genotype at SLC30A8 could predict baseline proinsulin levels independently of insulin levels [122]. The SLC30A8 rs13266634 (C allele) variant was associated with rosiglitazone therapy in HOMA-B, which is a homeostasis index assessing insulin secretion and $\beta$ cell function. The $\mathrm{C}$ allele carriers showed a more active response to the drug [123].

ZnT8 has been confirmed to play an important role in maintaining glucose hemostasis. In human studies, carriers of the risk allele of SLC30A8 have shown decreased insulin secretion, reduced proinsulin to insulin conversion, less insulin sensitivity, impaired glucose metabolism, and diminished $\beta$ cell function. Results from animal studies were inconsistent, but these conflicting results may be due to inconsistent animal species, various KO methods, and/or different diet and other environmental conditions. Some important outcomes should be highlighted. Under normal condition, ZnT8 did not show a significant function phenotype, but it played an important role in the high-fat diet condition [106]. These show that gene and environmental interactions contribute to disease development as it is known in human T1DM. ZnT8 is highly sensitive to unbalanced glucose conditions, and both $\alpha$ and $\beta$ cells contribute to glucose control in the whole body. Therefore, further investigation of ZnT8 function should be holistic and should include a broad panel of tests in response to the dynamic change of glucose. In our previous results, we found the $\mathrm{ZnT} 8$ risk mutation has increased functional activity, while loss-of-function mutations could protect from T2DM risk in humans; increased insulin secretion in animals was also reported. We showed that a membrane-embedded human ZnT8 antigen triggered a vigorous immune response in $\mathrm{ZnT} 8-\mathrm{KO}$ mice, and anti-ZnT8 autoantibodies which targeted the conformational (TM) epitopes could be beneficial to T1DM diagnosis. The conformational (TM) epitopes of ZnT8 are very important not only for detecting the cell surface ZnT8 expression, but also for function regulation by binding with cell surface 
ZnT8. Future studies are needed to clarify whether ZnT8 was blocked by anti-ZnT8 antibody effects on insulin secretion and whether it has a role for T2DM therapy.

\section{Conflicts of Interest}

The authors declare that they have no conflicts of interest.

\section{Acknowledgments}

This study was supported by the National Natural Science Foundation of China Grant Nos. 81673492 and 81202596.

\section{References}

[1] C. T. Chasapis, A. C. Loutsidou, C. A. Spiliopoulou, and M. E. Stefanidou, "Zinc and human health: an update," Archives of Toxicology, vol. 86, no. 4, pp. 521-534, 2012.

[2] T. Kambe, "Chapter eight. Molecular architecture and function of ZnT transporters," Current Topics in Membranes, vol. 69, pp. 199-220, 2012.

[3] S. A. Myers, A. Nield, and M. Myers, "Zinc transporters, mechanisms of action and therapeutic utility: implications for type 2 diabetes mellitus," Journal of Nutrition and Metabolism, vol. 2012, Article ID 173712, 13 pages, 2012.

[4] G. A. Rutter and F. Chimienti, "SLC30A8 mutations in type 2 diabetes," Diabetologia, vol. 58, no. 1, pp. 31-36, 2015.

[5] G. Dodson and D. Steiner, "The role of assembly in insulin's biosynthesis," Current Opinion in Structural Biology, vol. 8, no. 2, pp. 189-194, 1998.

[6] D. F. Steiner, Y. Rouillé, Q. Gong, S. Martin, R. Carroll, and S. J. Chan, "The role of prohormone convertases in insulin biosynthesis: evidence for inherited defects in their action in man and experimental animals," Diabetes \& Metabolism, vol. 22, no. 2, pp. 94-104, 1996.

[7] N. Havu, G. Lundgren, and S. Falkmer, "Zinc and manganese contents of micro-dissected pancreatic islets of some rodents. A microchemical study in adult and newborn guinea pigs, rats, Chinese hamsters and spiny mice," Acta Endocrinologica, vol. 86, no. 3, pp. 570-577, 1977.

[8] J. L. Vinkenborg, T. J. Nicolson, E. A. Bellomo, M. S. Koay, G. A. Rutter, and M. Merkx, "Genetically encoded FRET sensors to monitor intracellular $\mathrm{Zn}^{2+}$ homeostasis," Nature Methods, vol. 6, no. 10, pp. 737-740, 2009.

[9] Q. Huang, T. L. Yang, B. S. Tang et al., "Two novel functional single nucleotide polymorphisms of ADRB3 are associated with type 2 diabetes in the Chinese population," The Journal of Clinical Endocrinology and Metabolism, vol. 98, no. 7, pp. E1272-E1277, 2013.

[10] Q. Huang, L. F. Zhang, Y. Cheng et al., “Trp64Arg (rs4994) polymorphism of $\beta 3$-adrenergic receptor gene is associated with hyperuricemia in a Chinese male population," Clinical Chemistry and Laboratory Medicine, vol. 51, no. 9, pp. 1755-1760, 2013.

[11] Q. Huang, J. Y. Yin, X. P. Dai et al., "IGF2BP2 variations influence repaglinide response and risk of type 2 diabetes in Chinese population," Acta Pharmacologica Sinica, vol. 31, no. 6, pp. 709-717, 2010.

[12] T. Delong, T. A. Wiles, R. L. Baker et al., "Pathogenic CD4 T cells in type 1 diabetes recognize epitopes formed by peptide fusion," Science, vol. 351, no. 6274, pp. 711-714, 2016.
[13] M. Basaki, M. Saeb, S. Nazifi, and H. A. Shamsaei, "Zinc, copper, iron, and chromium concentrations in young patients with type 2 diabetes mellitus," Biological Trace Element Research, vol. 148, no. 2, pp. 161-164, 2012.

[14] A. El-Yazigi, N. Hannan, and D. A. Raines, "Effect of diabetic state and related disorders on the urinary excretion of magnesium and zinc in patients," Diabetes Research, vol. 22, no. 2, pp. 67-75, 1993.

[15] J. Jansen, E. Rosenkranz, S. Overbeck et al., "Disturbed zinc homeostasis in diabetic patients by in vitro and in vivo analysis of insulinomimetic activity of zinc," The Journal of Nutritional Biochemistry, vol. 23, no. 11, pp. 14581466, 2012.

[16] Q. Sun, R. M. van Dam, W. C. Willett, and F. B. Hu, "Prospective study of zinc intake and risk of type 2 diabetes in women," Diabetes Care, vol. 32, no. 4, pp. 629-634, 2009.

[17] I. Drake, G. Hindy, U. Ericson, and M. Orho-Melander, "A prospective study of dietary and supplemental zinc intake and risk of type 2 diabetes depending on genetic variation in SLC30A8," Genes \& Nutrition, vol. 12, no. 1, p. 30, 2017.

[18] F. Chimienti, S. Devergnas, A. Favier, and M. Seve, "Identification and cloning of a $\beta$-cell-specific zinc transporter, ZnT-8, localized into insulin secretory granules," Diabetes, vol. 53, no. 9, pp. 2330-2337, 2004.

[19] C. Cotsapas, L. Prokunina-Olsson, C. Welch et al., "Expression analysis of loci associated with type 2 diabetes in human tissues," Diabetologia, vol. 53, no. 11, pp. 2334-2339, 2010.

[20] R. Sladek, G. Rocheleau, J. Rung et al., "A genome-wide association study identifies novel risk loci for type 2 diabetes," Nature, vol. 445, no. 7130, pp. 881-885, 2007.

[21] D. S. Parsons, C. Hogstrand, and W. Maret, "The C-terminal cytosolic domain of the human zinc transporter ZnT8 and its diabetes risk variant," The FEBS Journal, vol. 285 , no. 7 , pp. 1237-1250, 2018.

[22] M. Lu, J. Chai, and D. Fu, "Structural basis for autoregulation of the zinc transporter YiiP," Nature Structural \& Molecular Biology, vol. 16, no. 10, pp. 1063-1067, 2009.

[23] M. Lu and D. Fu, "Structure of the zinc transporter YiiP," Science, vol. 317, no. 5845, pp. 1746-1748, 2007.

[24] A. Solomou, G. Meur, E. Bellomo et al., "The zinc transporter Slc30a8/ZnT8 is required in a subpopulation of pancreatic $\alpha$-cells for hypoglycemia-induced glucagon secretion," Journal of Biological Chemistry, vol. 290, no. 35, pp. 2143221442, 2015.

[25] K. Lemaire, F. Chimienti, and F. Schuit, "Zinc transporters and their role in the pancreatic $\beta$-cell," Journal of Diabetes Investigation, vol. 3, no. 3, pp. 202-211, 2012.

[26] S. Kleiner, D. Gomez, B. Megra et al., "Mice harboring the human SLC30A8 R138X loss-of-function mutation have increased insulin secretory capacity," Proceedings of the National Academy of Sciences of the United States of America, vol. 115, no. 32, pp. E7642-E7649, 2018.

[27] A. Segerstolpe, A. Palasantza, P. Eliasson et al., "Single-cell transcriptome profiling of human pancreatic islets in health and type 2 diabetes," Cell Metabolism, vol. 24, no. 4, pp. 593-607, 2016.

[28] L. D. Pound, Y. Hang, S. A. Sarkar et al., "The pancreatic islet $\beta$-cell-enriched transcription factor Pdx-1 regulates Slc30a8 gene transcription through an intronic enhancer," Biochemical Journal, vol. 433, no. 1, pp. 95-105, 2011. 
[29] L. Egefjord, J. L. Jensen, C. H. Bang-Berthelsen et al., "Zinc transporter gene expression is regulated by proinflammatory cytokines: a potential role for zinc transporters in beta-cell apoptosis?," BMC Endocrine Disorders, vol. 9, no. 1, p. 7, 2009.

[30] Q. Huang, C. Merriman, H. Zhang, and D. Fu, "Coupling of insulin secretion and display of a granule-resident zinc transporter ZnT8 on the surface of pancreatic beta cells," Journal of Biological Chemistry, vol. 292, no. 10, pp. 4034-4043, 2017.

[31] J. Hald, T. Galbo, C. Rescan et al., "Pancreatic islet and progenitor cell surface markers with cell sorting potential," Diabetologia, vol. 55, no. 1, pp. 154-165, 2012.

[32] G. A. Martens, V. de Punt, and G. Stangé, "CD99 as surface anchor for human islet endocrine cell purification," Journal of Tissue Engineering and Regenerative Medicine, vol. 12, no. 1, pp. e171-e176, 2018.

[33] S. Cauchi, C. Proença, H. Choquet et al., "Analysis of novel risk loci for type 2 diabetes in a general French population: the D.E.S.I.R. study," Journal of Molecular Medicine, vol. 86, no. 3, pp. 341-348, 2008.

[34] L. J. Scott, K. L. Mohlke, L. L. Bonnycastle et al., "A genome-wide association study of type 2 diabetes in Finns detects multiple susceptibility variants," Science, vol. 316, no. 5829, pp. 1341-1345, 2007.

[35] Diabetes Genetics Initiative of Broad Institute of Harvard and MIT, Lund University, and Novartis Institutes of BioMedical Research, R. Saxena, B. F. Voight et al., "Genome-wide association analysis identifies loci for type 2 diabetes and triglyceride levels," Science, vol. 316, no. 5829, pp. 1331-1336, 2007.

[36] E. Zeggini, M. N. Weedon, C. M. Lindgren et al., "Replication of genome-wide association signals in UK samples reveals risk loci for type 2 diabetes," Science, vol. 316, no. 5829, pp. 1336-1341, 2007.

[37] M. C. Y. Ng, K. S. Park, B. Oh et al., "Implication of genetic variants near TCF7L2, SLC30A8, HHEX, CDKAL1, $C D K N 2 A / B, I G F 2 B P 2$, and FTO in type 2 diabetes and obesity in 6,719 Asians," Diabetes, vol. 57, no. 8, pp. 22262233, 2008

[38] Y. Wu, H. Li, R. J. F. Loos et al., "Common variants in CDKAL1, CDKN2A/B, IGF2BP2, SLC30A8, and HHEX/IDE genes are associated with type 2 diabetes and impaired fasting glucose in a Chinese Han population," Diabetes, vol. 57, no. 10, pp. 2834-2842, 2008.

[39] C. Hu, R. Zhang, C. Wang et al., "PPARG, KCNJ11, CDKAL1, CDKN2A-CDKN2B, IDE-KIF11-HHEX, IGF2BP2 and SLC30A8 are associated with type 2 diabetes in a Chinese population," PLoS One, vol. 4, no. 10, article e7643, 2009.

[40] X. Han, Y. Luo, Q. Ren et al., "Implication of genetic variants near SLC30A8, HHEX, CDKAL1, CDKN2A/B, IGF2BP2, FTO, TCF2, KCNQ1, and WFS1 in type 2 diabetes in a Chinese population," BMC Medical Genetics, vol. 11, no. 1, p. 81,2010 .

[41] M. Xu, Y. Bi, Y. Xu et al., "Combined effects of 19 common variations on type 2 diabetes in Chinese: results from two community-based studies," PLoS One, vol. 5, no. 11, article e14022, 2010.

[42] J. Xu, J. Wang, and B. Chen, "SLC30A8 (ZnT8) variations and type 2 diabetes in the Chinese Han population," Genetics and Molecular Research, vol. 11, no. 2, pp. 1592-1598, 2012.

[43] F. J. Wei, C. Y. Cai, P. Yu et al., "Quantitative candidate gene association studies of metabolic traits in Han Chinese type 2 diabetes patients," Genetics and Molecular Research, vol. 14, no. 4, pp. 15471-15481, 2015.

[44] J. Liu, L. Wang, Y. Qian et al., "Association of 48 type 2 diabetes susceptibility loci with fasting plasma glucose and lipid levels in Chinese Hans," Diabetes Research and Clinical Practice, vol. 139, pp. 114-121, 2018.

[45] S. Omori, Y. Tanaka, A. Takahashi et al., "Association of CDKAL1, IGF2BP2, CDKN2A/B, HHEX, SLC30A8, and $K C N J 11$ with susceptibility to type 2 diabetes in a Japanese population," Diabetes, vol. 57, no. 3, pp. 791-795, 2008.

[46] F. Takeuchi, M. Serizawa, K. Yamamoto et al., "Confirmation of multiple risk loci and genetic impacts by a genome-wide association study of type 2 diabetes in the Japanese population," Diabetes, vol. 58, no. 7, pp. 1690-1699, 2009.

[47] Y. Horikawa, K. Miyake, K. Yasuda et al., "Replication of genome-wide association studies of type 2 diabetes susceptibility in Japan," The Journal of Clinical Endocrinology \& Metabolism, vol. 93, no. 8, pp. 3136-3141, 2008.

[48] Y. Tabara, H. Osawa, R. Kawamoto et al., "Replication study of candidate genes associated with type 2 diabetes based on genome-wide screening," Diabetes, vol. 58, no. 2, pp. $493-$ 498, 2009.

[49] J. T. Tan, D. P. K. Ng, S. Nurbaya et al., "Polymorphisms identified through genome-wide association studies and their associations with type 2 diabetes in Chinese, Malays, and Asian-Indians in Singapore," The Journal of Clinical Endocrinology \& Metabolism, vol. 95, no. 1, pp. 390-397, 2010.

[50] Y. M. Cho, T. H. Kim, S. Lim et al., "Type 2 diabetes-associated genetic variants discovered in the recent genome-wide association studies are related to gestational diabetes mellitus in the Korean population," Diabetologia, vol. 52, no. 2, pp. 253-261, 2009.

[51] Y. H. Lee, E. S. Kang, S. H. Kim et al., "Association between polymorphisms in SLC30A8, HHEX, CDKN2A/B, IGF2BP2, FTO, WFS1, CDKAL1, KCNQ1 and type 2 diabetes in the Korean population," Journal of Human Genetics, vol. 53, no. 11-12, pp. 991-998, 2008.

[52] N. Mtiraoui, A. Turki, R. Nemr et al., "Contribution of common variants of ENPP1, IGF2BP2, KCNJ11, MLXIPL, PPAR $\gamma, S L C 30 A 8$ and TCF7L2 to the risk of type 2 diabetes in Lebanese and Tunisian Arabs," Diabetes \& Metabolism, vol. 38, no. 5, pp. 444-449, 2012.

[53] J. K. Hertel, S. Johansson, H. Ræder et al., "Genetic analysis of recently identified type 2 diabetes loci in 1,638 unselected patients with type 2 diabetes and 1,858 control participants from a Norwegian population-based cohort (the HUNT study)," Diabetologia, vol. 51, no. 6, pp. 971-977, 2008.

[54] S. M. Ruchat, C. E. Elks, R. J. F. Loos et al., "Association between insulin secretion, insulin sensitivity and type 2 diabetes susceptibility variants identified in genome-wide association studies," Acta Diabetologica, vol. 46, no. 3, pp. 217-226, 2009.

[55] E. Ramos, G. Chen, D. Shriner et al., "Replication of genome-wide association studies (GWAS) loci for fasting plasma glucose in African-Americans," Diabetologia, vol. 54, no. 4, pp. 783-788, 2011.

[56] S. D. Rees, M. Z. I. Hydrie, A. S. Shera et al., "Replication of 13 genome-wide association (GWA)-validated risk variants for type 2 diabetes in Pakistani populations," Diabetologia, vol. 54, no. 6, pp. 1368-1374, 2011. 
[57] C. Kifagi, K. Makni, M. Boudawara et al., "Association of genetic variations in TCF7L2, SLC30A8, HHEX, LOC387761, and EXT2 with type 2 diabetes mellitus in Tunisia," Genetic Testing and Molecular Biomarkers, vol. 15, no. 6, pp. 399-405, 2011.

[58] M. A. Gamboa-Melendez, A. Huerta-Chagoya, H. MorenoMacias et al., "Contribution of common genetic variation to the risk of type 2 diabetes in the Mexican mestizo population," Diabetes, vol. 61, no. 12, pp. 3314-3321, 2012.

[59] N. M. Al-Daghri, K. M. Alkharfy, M. S. Alokail et al., "Assessing the contribution of 38 genetic loci to the risk of type 2 diabetes in the Saudi Arabian population," Clinical Endocrinology, vol. 80, no. 4, pp. 532-537, 2014.

[60] M. D. Bazzi, F. A. Nasr, M. S. Alanazi et al., "Association between FTO, MC4R, SLC30A8, and KCNQ1 gene variants and type 2 diabetes in Saudi population," Genetics and Molecular Research, vol. 13, no. 4, pp. 10194-10203, 2014.

[61] N. Sikhayeva, A. Iskakova, N. Saigi-Morgui, E. Zholdybaeva, C. B. Eap, and E. Ramanculov, "Association between 28 single nucleotide polymorphisms and type 2 diabetes mellitus in the Kazakh population: a case-control study," BMC Medical Genetics, vol. 18, no. 1, p. 76, 2017.

[62] H. Faghih, S. R. Khatami, N. Azarpira, and A. M. Foroughmand, "SLC30A8 gene polymorphism (rs13266634 $\mathrm{C} / \mathrm{T}$ ) and type 2 diabetes mellitus in south Iranian population," Molecular Biology Reports, vol. 41, no. 5, pp. 2709-2715, 2014.

[63] J. C. Lara-Riegos, M. G. Ortiz-López, B. I. Peña-Espinoza et al., "Diabetes susceptibility in Mayas: evidence for the involvement of polymorphisms in HHEX, HNF4 $\alpha$, KCNJ11, PPAR, , CDKN2A/2B, SLC30A8, CDC123/CAMK1D, TCF7L2, $A B C A 1$ and SLC16A11 genes," Gene, vol. 565, no. 1, pp. 6875, 2015.

[64] N. Plengvidhya, C. Chanprasert, N. Chongjaroen, P. T. Yenchitsomanus, M. Homsanit, and W. Tangjittipokin, "Impact of KCNQ1, CDKN2A/2B, CDKAL1, HHEX, MTNR1B, SLC30A8, TCF7L2, and UBE2E2 on risk of developing type 2 diabetes in Thai population," BMC Medical Genetics, vol. 19, no. 1, p. 93, 2018.

[65] C. Votsi, C. Toufexis, K. Michailidou et al., "Type 2 diabetes susceptibility in the Greek-Cypriot population: replication of associations with TCF7L2, FTO, HHEX, SLC30A8 and IGF2BP2 polymorphisms," Genes, vol. 8, no. 1, p. 16, 2017.

[66] S. Cauchi, S. D. Guerra, H. Choquet et al., "Meta-analysis and functional effects of the SLC30A8 rs13266634 polymorphism on isolated human pancreatic islets," Molecular Genetics and Metabolism, vol. 100, no. 1, pp. 77-82, 2010.

[67] Y. L. Jing, Q. M. Sun, Y. Bi, S. M. Shen, and D. L. Zhu, "SLC30A8 polymorphism and type 2 diabetes risk: evidence from 27 study groups," Nutrition, Metabolism, and Cardiovascular Diseases, vol. 21, no. 6, pp. 398-405, 2011.

[68] K. Xu, M. Zha, X. Wu et al., "Association between rs13266634 $\mathrm{C} / \mathrm{T}$ polymorphisms of solute carrier family 30 member 8 (SLC30A8) and type 2 diabetes, impaired glucose tolerance, type 1 diabetes-a meta-analysis," Diabetes Research and Clinical Practice, vol. 91, no. 2, pp. 195-202, 2011.

[69] R. J. Strawbridge, J. Dupuis, I. Prokopenko et al., "Genomewide association identifies nine common variants associated with fasting proinsulin levels and provides new insights into the pathophysiology of type 2 diabetes," Diabetes, vol. 60, no. 10, pp. 2624-2634, 2011.
[70] Y. C. Chang, P. H. Liu, Y. H. Yu et al., "Validation of type 2 diabetes risk variants identified by genome-wide association studies in Han Chinese population: a replication study and meta-analysis," PLoS One, vol. 9, no. 4, article e95045, 2014.

[71] K. W. Hong, M. Chung, and S. B. Cho, "Meta-analysis of genome-wide association study of homeostasis model assessment $\beta$ cell function and insulin resistance in an east Asian population and the European results," Molecular Genetics and Genomics, vol. 289, no. 6, pp. 1247-1255, 2014.

[72] L. Cheng, D. Zhang, L. Zhou, J. Zhao, and B. Chen, "Association between SLC30A8 rs13266634 polymorphism and type 2 diabetes risk: a meta-analysis," Medical Science Monitor, vol. 21, pp. 2178-2189, 2015.

[73] M. Fan, W. Li, L. Wang et al., "Association of SLC30A8 gene polymorphism with type 2 diabetes, evidence from 46 studies: a meta-analysis," Endocrine, vol. 53, no. 2, pp. 381-394, 2016.

[74] Y. Y. Li, X. Z. Lu, H. Wang et al., "Solute carrier family 30 member 8 gene $807 \mathrm{C} / \mathrm{T}$ polymorphism and type 2 diabetes mellitus in the Chinese population: a meta-analysis including 6,942 subjects," Frontiers in Endocrinology, vol. 9, p. $263,2018$.

[75] A. G. Nikitin, V. Y. Potapov, O. I. Brovkina et al., "Association of polymorphic markers of genes FTO, KCNJ11, CDKAL1, SLC3OA8, and CDKN2B with type 2 diabetes mellitus in the Russian population," PeerJ, vol. 5, article e3414, 2017.

[76] N. M. Phani, P. Adhikari, S. K. Nagri, S. C. D’Souza, K. Satyamoorthy, and P. S. Rai, "Replication and relevance of multiple susceptibility loci discovered from genome wide association studies for type 2 diabetes in an Indian population," PLoS One, vol. 11, no. 6, article e0157364, 2016.

[77] G. A. Walford, T. Green, B. Neale et al., "Common genetic variants differentially influence the transition from clinically defined states of fasting glucose metabolism," Diabetologia, vol. 55, no. 2, pp. 331-339, 2012.

[78] C. Hu, R. Zhang, C. Wang et al., "Variants from GIPR, TCF7L2, DGKB, MADD, CRY2, GLIS3, PROX1, SLC30A8 and IGF1 are associated with glucose metabolism in the Chinese," PLoS One, vol. 5, no. 11, article e15542, 2010.

[79] L. M. 't Hart, A. M. Simonis-Bik, G. Nijpels et al., "Combined risk allele score of eight type 2 diabetes genes is associated with reduced first-phase glucose-stimulated insulin secretion during hyperglycemic clamps," Diabetes, vol. 59, no. 1, pp. 287-292, 2010.

[80] C. Kelliny, U. Ekelund, L. B. Andersen et al., "Common genetic determinants of glucose homeostasis in healthy children: the European Youth Heart Study," Diabetes, vol. 58, no. 12, pp. 2939-2945, 2009.

[81] A. Stancakova, T. Kuulasmaa, J. Paananen et al., “Association of 18 confirmed susceptibility loci for type 2 diabetes with indices of insulin release, proinsulin conversion, and insulin sensitivity in 5,327 nondiabetic Finnish men," Diabetes, vol. 58, no. 9, pp. 2129-2136, 2009.

[82] J. Xiang, X. Y. Li, M. Xu et al., "Zinc transporter-8 gene (SLC30A8) is associated with type 2 diabetes in Chinese," The Journal of Clinical Endocrinology and Metabolism, vol. 93, no. 10, pp. 4107-4112, 2008.

[83] K. Kirchhoff, F. Machicao, A. Haupt et al., "Polymorphisms in the TCF7L2, CDKAL1 and SLC3OA8 genes are associated with impaired proinsulin conversion," Diabetologia, vol. 51, no. 4, pp. 597-601, 2008. 
[84] A. Haupt, M. Guthoff, S. A. Schäfer et al., "The inhibitory effect of recent type 2 diabetes risk loci on insulin secretion is modulated by insulin sensitivity," The Journal of Clinical Endocrinology \& Metabolism, vol. 94, no. 5, pp. 17751780, 2009.

[85] A. S. Dimas, V. Lagou, A. Barker et al., "Impact of type 2 diabetes susceptibility variants on quantitative glycemic traits reveals mechanistic heterogeneity," Diabetes, vol. 63, no. 6, pp. 2158-2171, 2014.

[86] Y. Lin, P. Li, L. Cai et al., "Association study of genetic variants in eight genes/loci with type 2 diabetes in a Han Chinese population," BMC Medical Genetics, vol. 11, no. 1, p. 97, 2010.

[87] Q. Huang, J. Y. Yin, X. P. Dai et al., “Association analysis of SLC30A8 rs13266634 and rs16889462 polymorphisms with type 2 diabetes mellitus and repaglinide response in Chinese patients," European Journal of Clinical Pharmacology, vol. 66, no. 12, pp. 1207-1215, 2010.

[88] H. W. Davidson, J. M. Wenzlau, and R. M. O’Brien, “Zinc transporter 8 (ZnT8) and $\beta$ cell function," Trends in Endocrinology and Metabolism, vol. 25, no. 8, pp. 415-424, 2014.

[89] N. A. Seman, W. N. W. Mohamud, C. G. Östenson, K. Brismar, and H. F. Gu, "Increased DNA methylation of the SLC30A8 gene promoter is associated with type 2 diabetes in a Malay population," Clinical Epigenetics, vol. 7, no. 1, p. 30, 2015.

[90] I. A. Khan, S. Poornima, P. Jahan, P. Rao, and Q. Hasan, "Type 2 diabetes mellitus and the association of candidate genes in Asian Indian population from Hyderabad, India," Journal of Clinical and Diagnostic Research, vol. 9, no. 11, pp. GC01-GC05, 2015.

[91] G. Chauhan, C. J. Spurgeon, R. Tabassum et al., "Impact of common variants of PPARG, KCNJ11, TCF7L2, SLC30A8, HHEX, CDKN2A, IGF2BP2, and CDKAL1 on the risk of type 2 diabetes in 5,164 Indians," Diabetes, vol. 59, no. 8, pp. 2068-2074, 2010.

[92] U. J. Kommoju, J. Maruda, S. Kadarkarai et al., "No detectable association of IGF2BP2 and SLC30A8 genes with type 2 diabetes in the population of Hyderabad, India," Meta Gene, vol. 1, pp. 15-23, 2013.

[93] H. Kulkarni, M. Mamtani, J. M. Peralta et al., "Lack of association between SLC30A8 variants and type 2 diabetes in Mexican American families," Journal Diabetes Research, vol. 2016, Article ID 6463214, 9 pages, 2016.

[94] J. P. Lewis, N. D. Palmer, P. J. Hicks et al., "Association analysis in African Americans of European-derived type 2 diabetes single nucleotide polymorphisms from wholegenome association studies," Diabetes, vol. 57, no. 8, pp. 2220-2225, 2008.

[95] Go-T2D Consortium, T2D-GENES Consortium, J. Flannick et al., "Loss-of-function mutations in SLC30A8 protect against type 2 diabetes," Nature Genetics, vol. 46, no. 4, pp. 357-363, 2014.

[96] Y. Fu, W. Tian, E. B. Pratt et al., "Down-regulation of ZnT8 expression in INS-1 rat pancreatic beta cells reduces insulin content and glucose-inducible insulin secretion," PLoS One, vol. 4, no. 5, article e5679, 2009.

[97] N. Wijesekara, F. F. Dai, A. B. Hardy et al., "Beta cell-specific Znt8 deletion in mice causes marked defects in insulin processing, crystallisation and secretion," Diabetologia, vol. 53, no. 8, pp. 1656-1668, 2010.
[98] T. J. Nicolson, E. A. Bellomo, N. Wijesekara et al., "Insulin storage and glucose homeostasis in mice null for the granule zinc transporter ZnT8 and studies of the type 2 diabetesassociatedvariants," Diabetes, vol. 58, no. 9, pp. 20702083, 2009.

[99] K. Lemaire, M. A. Ravier, A. Schraenen et al., "Insulin crystallization depends on zinc transporter ZnT8 expression, but is not required for normal glucose homeostasis in mice," Proceedings of the National Academy of Sciences of the United States of America, vol. 106, no. 35, pp. 14872-14877, 2009.

[100] R. K. Mitchell, M. Hu, P. L. Chabosseau et al., "Molecular genetic regulation of Slc30a8/ZnT8 reveals a positive association with glucose tolerance," Molecular Endocrinology, vol. 30, no. 1, pp. 77-91, 2016.

[101] L. D. Pound, S. A. Sarkar, A. Ustione et al., "The physiological effects of deleting the mouse SLC30A8 gene encoding zinc transporter- 8 are influenced by gender and genetic background," PLoS One, vol. 7, no. 7, article e40972, 2012.

[102] L. D. Pound, S. A. Sarkar, R. K. P. Benninger et al., "Deletion of the mouse Slc30a8 gene encoding zinc transporter-8 results in impaired insulin secretion," Biochemical Journal, vol. 421, no. 3, pp. 371-376, 2009.

[103] M. Tamaki, Y. Fujitani, A. Hara et al., "The diabetessusceptible gene SLC30A8/ZnT8 regulates hepatic insulin clearance," The Journal of Clinical Investigation, vol. 123, no. 10, pp. 4513-4524, 2013.

[104] L. Li, S. Bai, and C. T. Sheline, "hZnT8 (Slc30a8) transgenic mice that overexpress the R325W polymorph have reduced islet $\mathrm{Zn}^{2+}$ and proinsulin levels, increased glucose tolerance after a high-fat diet, and altered levels of pancreatic zinc binding proteins," Diabetes, vol. 66, no. 2, pp. 551-559, 2017.

[105] K. E. Syring, K. A. Boortz, J. K. Oeser et al., "Combined deletion of Slc30a7 and Slc30a8 unmasks a critical role for ZnT8 in glucose-stimulated insulin secretion," Endocrinology, vol. 157, no. 12, pp. 4534-4541, 2016.

[106] A. B. Hardy, N. Wijesekara, I. Genkin et al., "Effects of high-fat diet feeding on Znt8-null mice: differences between $\beta$-cell and global knockout of Znt8," American Journal of Physiology-Endocrinology and Metabolism, vol. 302, no. 9, pp. E1084-E1096, 2012.

[107] A. Solomou, E. Philippe, P. Chabosseau et al., "Over-expression of Slc30a8/ZnT8 selectively in the mouse $\alpha$ cell impairs glucagon release and responses to hypoglycemia," Nutrition \& Metabolism, vol. 13, no. 1, p. 46, 2016.

[108] J. M. Wenzlau, K. Juhl, L. Yu et al., “The cation efflux transporter ZnT8 (Slc30A8) is a major autoantigen in human type 1 diabetes," Proceedings of the National Academy of Sciences of the United States of America, vol. 104, no. 43, pp. 1704017045, 2007.

[109] C. Ploix, I. Bergerot, N. Fabien, S. Perche, V. Moulin, and C. Thivolet, "Protection against autoimmune diabetes with oral insulin is associated with the presence of IL- 4 type 2 T-cells in the pancreas and pancreatic lymph nodes," Diabetes, vol. 47, no. 1, pp. 39-44, 1998.

[110] B. Yi, G. Huang, and Z. Zhou, "Different role of zinc transporter 8 between type 1 diabetes mellitus and type 2 diabetes mellitus," Journal of Diabetes Investigation, vol. 7, no. 4, pp. 459-465, 2016.

[111] B. Yi, G. Huang, and Z. G. Zhou, "Current and future clinical applications of Zinc Transporter-8 in type 1 diabetes mellitus," Chinese Medical Journal, vol. 128, no. 17, pp. 23872394, 2015. 
[112] Y. Xiang, G. Huang, Y. Zhu et al., "Identification of autoimmune type 1 diabetes and multiple organ-specific autoantibodies in adult-onset non-insulin requiring diabetes in China: a population based multicenter national wide survey," Diabetes, Obesity and Metabolism, 2018.

[113] J. M. Wenzlau, J. C. Hutton, and H. W. Davidson, "New antigenic targets in type 1 diabetes," Current Opinion in Endocrinology, Diabetes, and Obesity, vol. 15, no. 4, pp. 315-320, 2008.

[114] J. M. Wenzlau, O. Moua, Y. Liu, G. S. Eisenbarth, J. C. Hutton, and H. W. Davidson, "Identification of a major humoral epitope in Slc30A8 (ZnT8)," Annals of the New York Academy of Sciences, vol. 1150, no. 1, pp. 252-255, 2008.

[115] D. A. Chistiakov and N. V. Voronova, " $\mathrm{Zn}(2+)$-transporter-8: a dual role in diabetes," BioFactors, vol. 35 , no. 4 , pp. 356-363, 2009.

[116] T. Kimpimäki, A. Kupila, A. M. Hämäläinen et al., “The first signs of $\beta$-cell autoimmunity appear in infancy in genetically susceptible children from the general population: the Finnish Type 1 Diabetes Prediction and Prevention Study," The Journal of Clinical Endocrinology and Metabolism, vol. 86, no. 10, pp. 4782-4788, 2001.

[117] J. M. M. Howson, S. Krause, H. Stevens et al., "Genetic association of zinc transporter 8 ( $\mathrm{ZnT}$ ) autoantibodies in type 1 diabetes cases," Diabetologia, vol. 55, no. 7, pp. 19781984, 2012.

[118] H. Wan, C. Merriman, M. A. Atkinson et al., "Proteoliposome-based full-length ZnT8 self-antigen for type 1 diabetes diagnosis on a plasmonic platform," Proceedings of the National Academy of Sciences of the United States of America, vol. 114, no. 38, pp. 10196-10201, 2017.

[119] E. Kawasaki, M. Uga, K. Nakamura et al., "Association between anti-ZnT8 autoantibody specificities and SLC30A8 Arg325Trp variant in Japanese patients with type 1 diabetes," Diabetologia, vol. 51, no. 12, pp. 2299-2302, 2008.

[120] C. Merriman, Q. Huang, W. Gu, L. Yu, and D. Fu, “A subclass of serum anti-ZnT8 antibodies directed to the surface of live pancreatic $\beta$-cells," Journal of Biological Chemistry, vol. 293, no. 2, pp. 579-587, 2018.

[121] C. Merriman, H. Li, H. Li, and D. Fu, "Highly specific monoclonal antibodies for allosteric inhibition and immunodetection of the human pancreatic zinc transporter ZnT8," Journal of Biological Chemistry, vol. 293, no. 42, pp. 1620616216, 2018.

[122] for the DPP Research Group, A. R. Majithia, K. A. Jablonski et al., "Association of the SLC30A8 missense polymorphism $\mathrm{R} 325 \mathrm{~W}$ with proinsulin levels at baseline and after lifestyle, metformin or troglitazone intervention in the Diabetes Prevention Program," Diabetologia, vol. 54, no. 10, pp. 25702574, 2011.

[123] F. Jiang, Q. Li, C. Hu et al., "Association of a SLC30A8 genetic variant with monotherapy of repaglinide and rosiglitazone effect in newly diagnosed type 2 diabetes patients in China," Biomedical and Environmental Sciences, vol. 25, no. 1, pp. 23-29, 2012. 


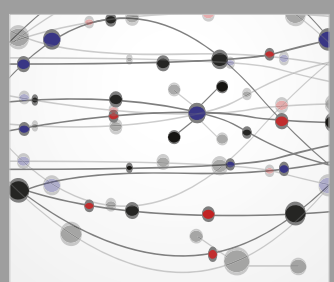

The Scientific World Journal
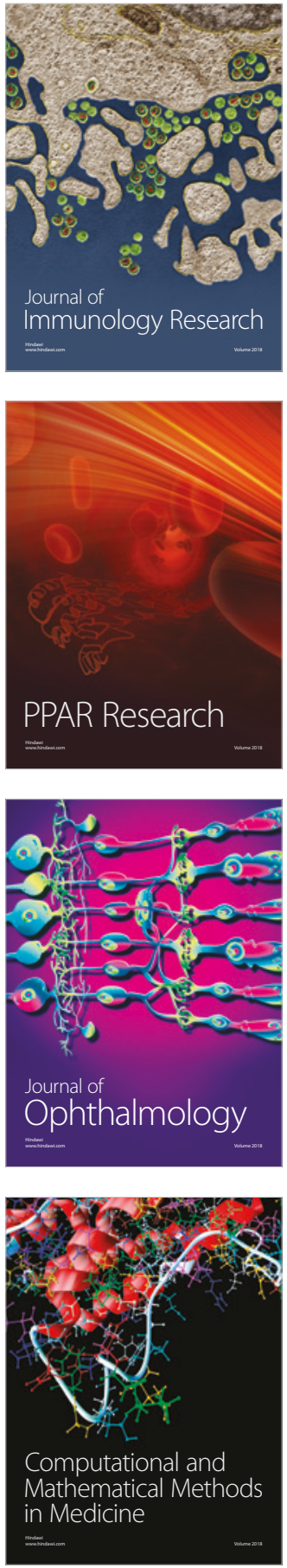

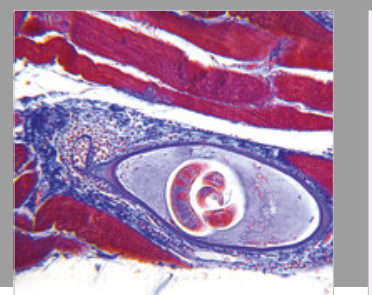

Gastroenterology Research and Practice

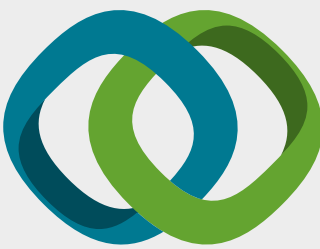

\section{Hindawi}

Submit your manuscripts at

www.hindawi.com
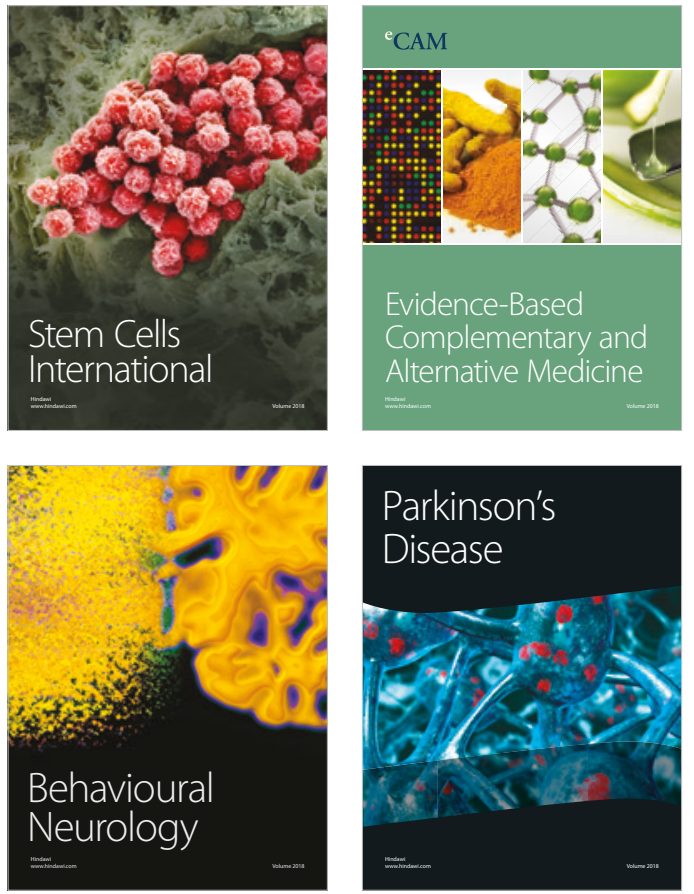

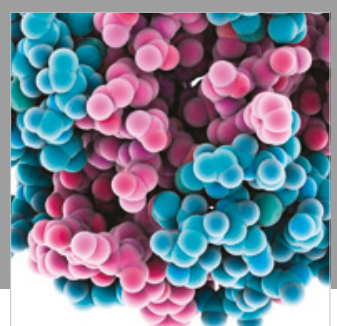

ournal of

Diabetes Research

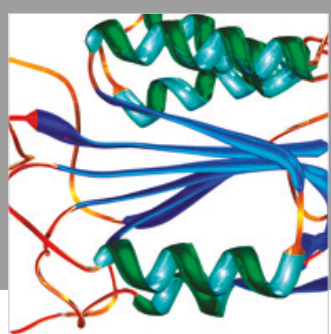

Disease Markers
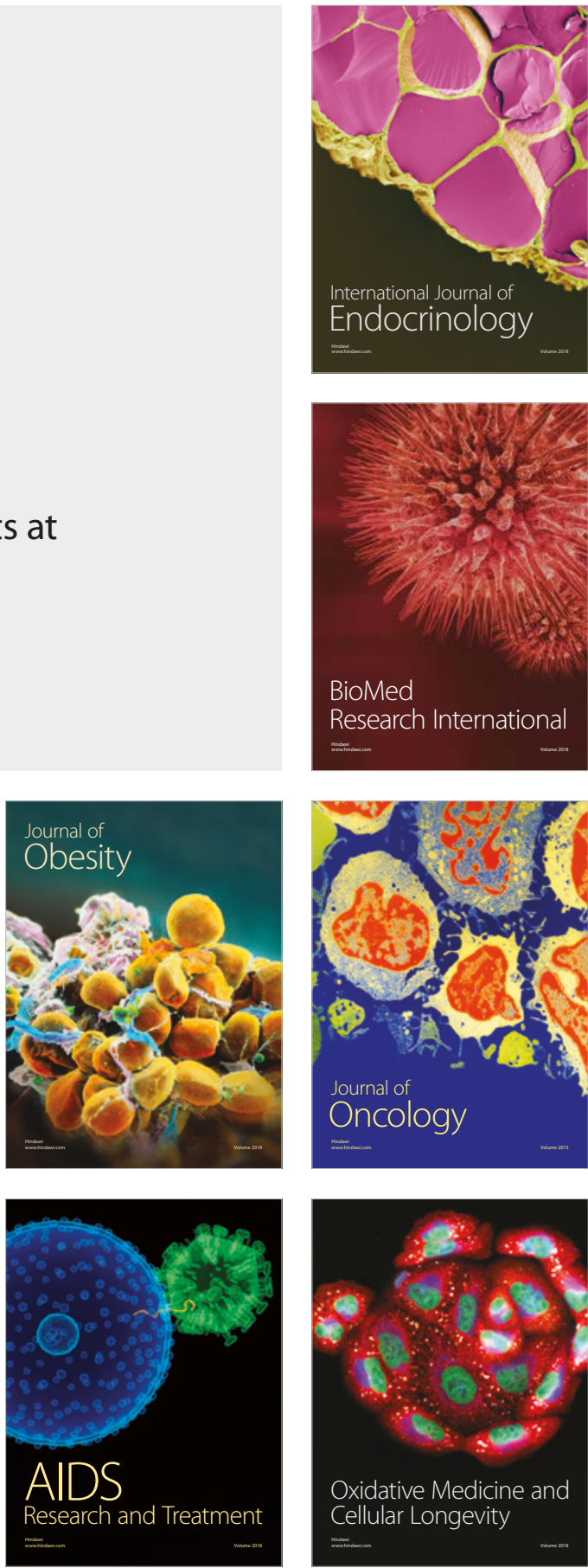